\title{
Efectos en la adherencia al tratamiento y en el ajuste psicológico luego de la revelación del diagnóstico de VIH/SIDA con el modelo clínico "DIRÉ" en niños y jóvenes colombianos menores de 17 años
}

\author{
Ana María Trejos, Lizeth Reyes, Marly Johana Bahamon, Yolima Alarcón y Gladys Gaviria
}

\footnotetext{
Facultad de Psicología de la

Universidad Simón Bolívar. Barranquilla-Colombia. Universidad del Norte (AMT, MJB, YA) Grupo de Investigación Desarrollo Humano, Educación y Procesos Sociales de la Universidad Simón Bolivar. Barranquilla-Colombia. Universidad del Norte (LR, YA) Universidad de

Manizales-CINDE (MJB) Facultad de Enfermería de la Universidad Simón Bolívar Barranquilla-Colombia. Enfermera. Universidad Simón Bolívar (GG)

Ninguno de los autores presenta conflicto de intereses que declarar.

Establecimientos donde se realizó la investigación

Oncohivvida IPS, Medicina Integra IPS, Fundación Santo Tomás de Villanueva IPS, IPS de la Costa y Saludcoop EPS

Proyecto de Investigación ganador de una beca para estudios de Doctorado obtenida en la I Convocatoria para estudios de Doctorado del Departamento

Investigación, Desarrollo e Innovación (DIDI) de la Universidad del Norte (2008).

Recibido: 20 de noviembre de 2014 Aceptado: 20 de julio de 2015

Correspondencia a: Ana María Trejos Herrera atrejos1@unisimonbolivar.edu.co
}

\section{Introducción}

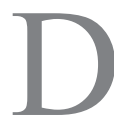
e acuerdo a los resultados de un estudio realizado en población infantil afectada por VIH/SIDA en cinco ciudades colombianas, sólo 3,8\% (n: 11) de los niños y niñas tenían conocimiento de su diagnóstico de infección por $\mathrm{VIH} / \mathrm{SIDA}^{1}$; las razones para retrasar la entrega del diagnóstico, reportadas por profesionales de salud y cuidadores de los menores afectados [96,\% (n: 275)] se relacionan con la protección de los menores frente al daño psicológico o estrés emocional y falta de capacitación respecto al procedimiento y edad para entregar esta información. Los anteriores resultados indican que en el contexto colombiano, la revelación del diagnóstico de infección por VIH a los niños y niñas no es una práctica consolidada ${ }^{2}$.
Especial atención han recibido las directrices de la Academia Americana de Pediatría sobre la revelación de la infección por VIH; estas directrices sostienen que todos los adolescentes deben conocer su diagnóstico y que la revelación debe ser considerada para niños en edad escolar teniendo en cuenta su edad, nivel de desarrollo cognitivo, madurez psicosocial, complejidad de la dinámica familiar y el contexto clínico ${ }^{3}$.

Mantener el secreto es una estrategia frecuentemente usada por las familias afectadas debido al estigma asociado a la infección por VIH/SIDA y a sus formas de transmisión ${ }^{4,5}$. Spinetta \& Deasy-Spinetta ${ }^{6}$ sostienen que a pesar de los esfuerzos que los padres realizan para ocultar el diagnóstico, los niños suelen llegar a conocerlo aunque nunca se lo hayan dicho expresamente. Este fenómeno ha sido reportado en la literatura médica sobre los niños 
con otras condiciones que amenazan la vida y, sugiere que probablemente es imposible ocultar el diagnóstico indefinidamente a los niños.

Con el desarrollo, se considera adecuado que niños mayores y adolescentes sean conscientes de su diagnóstico a fin de que, con la educación y apoyo emocional, puedan llegar a tener más control sobre el proceso de su enfermedad. Para los adolescentes se considera aún más imperativo para no exponer a otros, sin saberlo, durante la actividad sexual ${ }^{7}$. De igual manera, para alcanzar una mejor calidad de vida se debe tener un diagnóstico oportuno, tratamiento eficaz, apoyo físico, emocional, psicológico, social y sobre todo, el poder recibir ayuda en momentos de desamparo ${ }^{8}$.

Pese a los temores reportados por las familias afectadas por el VIH/SIDA, la literatura científica muestra que comunicar el diagnóstico puede tener importantes beneficios: promueve una discusión abierta y honesta entre adultos y niños; promueve el crecimiento intelectual, emocional y la oportunidad de ayudar al niño a hacer frente a la enfermedad y manejar emociones profundas. Los padres de niños afectados con infección por VIH y SIDA que han revelado el diagnóstico a sus niños han declarado que se han comportado con más madurez y responsabilidad luego de aprender el diagnóstico ${ }^{21}$.

En este sentido, el objetivo de la presente investigación fue evaluar los efectos del modelo clínico "DIRÉ" de revelación del diagnóstico de VIH/SIDA en niños y niñas bajo 17 años de edad. Hipotéticamente tenía efectos en el mantenimiento de la adherencia al tratamiento anti-retroviral y en la normalización del ajuste psicológico, basado en el modelo clínico de revelación del diagnóstico de Blasini y otros ${ }^{9}$, proporcionando un referente en el contexto colombiano respecto a cómo debe realizarse esta importante comunicación y los beneficios de la misma en la aplicación de componentes de trabajo con profesionales que brindan servicios de salud, cuidadores y niños y niñas seropositivos para VIH/SIDA.

El modelo Blasini y otros ${ }^{9}$, fue diseñado e implementado en Puerto Rico por un equipo interdisciplinario de psicólogos, trabajadores sociales, enfermeras y pediatras (tanto generales como especialistas en el comportamiento y desarrollo de enfermedades infecciosas) de la Unidad de Investigación Clínica en SIDA Pediátrico (Proyecto GAMMA) en el Centro Cardiovascular de Puerto Rico y del Caribe, adscrito al recinto de ciencias médicas de la Universidad de Puerto Rico. El objetivo de este modelo, adecuado al desarrollo cognitivo de cada niño (a) con infección por VIH y centrado en la familia, es superar el estigma, la indefensión y empoderar a los menores afectados y a sus cuidadores ofreciéndoles opciones, información y apoyo mediante la promoción del respeto y sensibilidad frente a los valores culturales y al estilo de comunicación, función, estructura, los rituales y creencias religiosas de la familia.

El modelo "DIRÉ" toma los cinco componentes de base del modelo Blasini y otros ${ }^{9}$ e incorpora modificaciones en los mismos relacionadas con la incorporación de evaluaciones psicosociales y metodologías lúdicas para abordar el proceso de revelación con los menores.

\section{Materiales y Métodos}

\section{Participantes}

Mediante muestreo no probabilístico, por criterio o fines especiales, participaron 41 profesionales de la salud pertenecientes a cuatro instituciones prestadoras de servicios de salud (IPS) del régimen subsidiado y a una del régimen contributivo de la ciudad de Barranquilla; 31 cuidadores y, 33 pacientes bajo 17 años de edad, con infección por VIH/SIDA (Tablas 1-3). Las instituciones fueron contactadas mediante listado de servicios de Infectología de la Secretaría de Salud Distrital de Barranquilla.

El protocolo de investigación y el consentimiento informado obtuvieron el aval del Comité de Ética en Investigación en el área de la salud de la Decanatura de Salud de la Universidad del Norte así como de los respectivos Comités de Ética de las Instituciones participantes.

\section{Instrumentos}

Con base en la validación de instrumentos desarrollados en la población infantil afectada por VIH/SIDA en cinco ciudades colombianas ${ }^{2}$ se realizó un proceso de

Tabla 1. Estadísticas descriptivas de las variables socio-demográficas en profesionales de la salud pertenecientes a instituciones prestadoras de servicios de salud

de Barranquilla. Variables Socio-demográficas de profesionales de la salud ( $n: 41)$

\begin{tabular}{|c|c|c|}
\hline Variables & & Frecuencia \% \\
\hline Edad (años) & $\begin{array}{l}\leq 33 \\
34-47 \\
48 \text { o más }\end{array}$ & $\begin{array}{c}17(41,5 \%) \\
17(41,5 \%) \\
7(17 \%)\end{array}$ \\
\hline Sexo & $\begin{array}{l}\text { Masculino } \\
\text { Femenino }\end{array}$ & $\begin{array}{r}9(22 \%) \\
32(78 \%)\end{array}$ \\
\hline Profesión & $\begin{array}{l}\text { Médicos y Especialistas } \\
\text { Psicología } \\
\text { Trabajo Social } \\
\text { Enfermería y Auxiliares de Salud } \\
\text { Otros profesionales }\end{array}$ & $\begin{array}{l}8(19,5 \%) \\
8(19,5 \%) \\
8(19,5 \%) \\
9(22 \%) \\
8(19,5 \%)\end{array}$ \\
\hline Años de experiencia & $\begin{array}{l}\text { Poca experiencia ( } 1-2 \text { años) } \\
\text { Experiencia intermedia (3-4 años) } \\
\text { Mucha experiencia ( } 5 \text { o más años) }\end{array}$ & $\begin{array}{r}28(68,3 \%) \\
12(29,3 \%) \\
1 \quad(2,4 \%)\end{array}$ \\
\hline Condición laboral & $\begin{array}{l}\text { Empleado a tiempo completo } \\
\text { Empleado a tiempo parcial }\end{array}$ & $\begin{aligned} 40 & (97,6 \%) \\
1 & (2,4 \%)\end{aligned}$ \\
\hline Número de instituciones donde labora & $\begin{array}{l}1 \text { institución } \\
2 \text { instituciones } \\
3 \text { o más instituciones }\end{array}$ & $\begin{array}{r}28(68,3 \%) \\
10(24,4 \%) \\
3(7,3 \%)\end{array}$ \\
\hline
\end{tabular}


Tabla 2. Estadísticas descriptivas de las variables socio-demográficas de cuidadores de niños (as) seropositivos para VIH/SIDA.

Variables socio-demográficas ( $\mathrm{n}: \mathbf{3 0}$ )

\section{Variables}

\begin{tabular}{|c|c|c|}
\hline \multirow[t]{5}{*}{ Edad (años) } & $\leq 32$ & $7(23,3 \%)$ \\
\hline & $32-38$ & $5(16,7 \%)$ \\
\hline & $39-53$ & $7(23,3 \%)$ \\
\hline & $54-57$ & $5(16,7 \%)$ \\
\hline & 58 o más & $6(20 \%)$ \\
\hline \multirow[t]{2}{*}{ Sexo } & Masculino & $5(16,7 \%)$ \\
\hline & Femenino & $15(83,3 \%)$ \\
\hline \multirow[t]{6}{*}{ Parentesco } & Madre/padre biológicos & $14(46,7 \%)$ \\
\hline & Abuelos/bisabuelos & $6(20 \%)$ \\
\hline & Tíos (as) & $6(20 \%)$ \\
\hline & Madre/padre sustitutos & $2(6,7 \%)$ \\
\hline & Hermanos (as) & $1(3,3 \%)$ \\
\hline & Sin parentesco & $1(3,3 \%)$ \\
\hline \multirow[t]{5}{*}{ Escolaridad } & Sin estudios & $3(10 \%)$ \\
\hline & Estudios primarios & $11(36,7 \%)$ \\
\hline & Secundaria incompleta & $8(26,7 \%)$ \\
\hline & Secundaria completa & $6(20 \%)$ \\
\hline & Universitarios & $2(6,7 \%)$ \\
\hline \multirow[t]{5}{*}{ Estado civil } & Soltero (a) & $9(30 \%)$ \\
\hline & Casado (a) & $2(6,7 \%)$ \\
\hline & Unión libre & $10(33,3 \%)$ \\
\hline & Separado (a) & $2(6,7 \%)$ \\
\hline & Viudo (a) & $7(23,3 \%)$ \\
\hline \multirow[t]{4}{*}{ Condición laboral } & Empleado a tiempo completo & $2(6,7 \%)$ \\
\hline & Empleado a tiempo parcial & $1(3,3 \%)$ \\
\hline & Trabajador independiente & $9(30 \%)$ \\
\hline & Desempleado (a) & $18(60 \%)$ \\
\hline \multirow[t]{4}{*}{ Estrato socio-económico } & Estrato 1 (más bajo) & $17(56,7 \%)$ \\
\hline & Estrato 2 & $11(36,7 \%)$ \\
\hline & Estrato 3 & $1(3,3 \%)$ \\
\hline & Estrato 5 & $1(3,3 \%)$ \\
\hline \multirow{3}{*}{$\begin{array}{l}\text { Ingresos económicos } \\
\text { del hogar }\end{array}$} & Menos de un SMLV & $17(56,7 \%)$ \\
\hline & Un SMLV & $7(23,3 \%)$ \\
\hline & De dos a tres SMLV & $6(20 \%)$ \\
\hline \multirow{6}{*}{$\begin{array}{l}\text { Edad que considera adecuada } \\
\text { para la revelación }\end{array}$} & $\leq 5$ años & $1(3,3 \%)$ \\
\hline & 6-8 años & $2(6,7 \%)$ \\
\hline & 9-11 años & $11(36,7 \%)$ \\
\hline & 12-14 años & $10(33,3 \%)$ \\
\hline & 15-18 años & $3(10 \%)$ \\
\hline & No aplica & $3(10 \%)$ \\
\hline
\end{tabular}

Tabla 3. Estadísticas descriptivas de las variables socio-demográficas de niños (as) afectados por VIH/SIDA

Variables socio-demográficas en niños (as) (n: 31)

Frecuencia \%

Edad (años)

$\leq 8$ años
$9-10$
$11-12$

$8(25,8 \%)$

$8(25,8 \%)$

$8(25,8 \%)$

13 o más

$7(22,6 \%)$

Sexo

Masculino

$13(41,9 \%)$

Femenino

$18(58,1 \%)$

Conocimiento del diagnóstico

Todos los familiares

$15(48,4 \%)$

del niño (a) en la familia

Tipo de vínculo

Algunos familiares

$16(51,6 \%)$

Tipo de vinculo

Hogar sustituto

$2(6,5 \%)$

A cargo de la familia extendida

$15(48,4 \%)$

A cargo de la madre/padre

o ambos

$13(41,9 \%)$

Cuidador no familiar

$1(3,2 \%)$

Motivo del vínculo diferente al

Muerte de la madre

$6(19,4 \%)$

materno o paterno

Abandono del padre

$1(3,2 \%)$

Muerte de ambos padres

$10(32,3 \%)$

Víctima de violación

$1(3,2 \%)$

No aplica

$13(41,9 \%)$

Edad del niño cuando

$<1$

$4(12,9 \%)$

el vínculo (años)

$1-3$

$7(22,6 \%)$

4-6

$2(6,5 \%)$

7-9

$2(6,5 \%)$

10 o más

$3(9,7 \%)$

No aplica

$13(41,8 \%)$

Fecha de diagnóstico

Recuerda día/mes/año

$2(6,5 \%)$

Recuerda mes/año

$16(51,6 \%)$

Recuerda año

9 (29\%)

No recuerda la fecha de diagnóstico

$4(12,9 \%)$

Afiliación Régimen de salud del Contributivo

$3(9,7 \%)$

niño (a)

Subsidiado

$28(90,3 \%)$

Institución donde va el niño (a)

Oncovihvida IPS

$10(32,2 \%)$

a revisión médica

Medicina Integral IPS

$6(19,4 \%)$

Fundación Social Santo

Tomás de Villanueva

$4(12,9 \%)$

IPS de la Costa

$8(25,8 \%)$

Saludcoop

$3(9,7 \%)$

Número de instituciones donde Una institución

$15(48,3 \%)$

el niño (a) ha sido atendido

Dos instituciones

Tres instituciones

$7(22,6 \%)$

Cuatro instituciones 
validación de contenido por criterio de jueces expertos utilizando la metodología propuesta por Moriyama ${ }^{10}$. Se diseñó un cuestionario de 20 preguntas para evaluar los patrones de revelación y no revelación del diagnóstico de infección por VIH/ SIDA a menores en profesionales de la salud y cuidadores participantes. Dos meses antes de su participación en el modelo se administraron cuestionarios a los cuidadores: Apgar Familiar ${ }^{11}$, EuroQol (EQ $-5 \mathrm{D})^{12}$, Encuesta de Apoyo Social MOS ${ }^{13}$, cuestionario de información del tratamiento para el VIH/SIDA ${ }^{9}$ y la Lista de Síntomas del Niño CBCL/6-18 ${ }^{14}$ adaptado a población latina para evaluar el ajuste psicológico dos meses antes y dos semanas, tres y seis meses después de la revelación del diagnóstico a los niños.

La información de las características clínicas de los niños con infección por VIH/SIDA se recogió en las historias clínicas de las instituciones involucradas en el estudio. Dos meses antes de su participación en el modelo, se administraron cuestionarios a los niños con infección por VIH/SIDA: Cuestionario pre-revelación del diagnóstico de $\mathrm{VIH}^{9}$, Cuestionario KINDL de calidad de vida relacionada con la salud ${ }^{15}$, Escala de auto-concepto de Piers-Harris ${ }^{16}$, Cuestionario de depresión infantil ${ }^{17}$, Cuestionario de ansiedad estado-rasgo $\mathrm{STAIC}^{18}$, Inventario de comportamiento de niños YSR/11-18 ${ }^{14}$, Test de Morisky-Green-Levine ${ }^{19}$ y el Test de Haynes-Sackett ${ }^{20}$. Los últimos tres cuestionarios se aplicaron además dos semanas, tres y seis meses después de la revelación del diagnóstico a los niños.

La adherencia al tratamiento para infección por $\mathrm{VIH} /$ SIDA fue medida con el Cuestionario de Adherencia al Tratamiento Auto-referido de Morisky-Green-Levine ${ }^{19}$ validado en versión española. Este cuestionario se considera como uno de los mejores métodos indirectos para medir el cumplimiento ya que presenta una alta especificidad y un alto valor predictor positivo, por lo cual puede ser una buena alternativa para identificar grupos de riesgo de no cumplimiento en atención primaria, ya que es un cuestionario corto, de fácil aplicación, y con escasos requisitos de nivel sociocultural para su comprensión. La medición que este test proporciona está considerada dentro de los métodos indirectos basados en la entrevista clínica para la evaluación de la adherencia al tratamiento.

El ajuste psicológico se evaluó con el Inventario del Comportamiento de Niños (as) de 6-18 años para PadresChild Behavior Checklist (CBCL). Este instrumento fue desarrollado por Achenbach y Rescorla ${ }^{14,21}$, y consta de dos apartados: el primero, permite obtener un perfil de competencias mediante un puntaje directo y un puntaje normativo o $\mathrm{T}$ en función de la edad y el sexo referido al desempeño del niño(a) según lo informado por los padres en diversas actividades y en su ámbito social y escolar, mientras que el segundo apartado evalúa la presencia de psicopatología en el niño (a) en los últimos seis meses, también según lo informado por los padres, con duración aproximada de $30 \mathrm{~min}$.

\section{Procedimiento}

Se utilizó un diseño cuasi-experimental de series temporales para evaluar los efectos del modelo clínico "DIRÉ" en cinco instituciones prestadoras de servicios de salud de la ciudad de Barranquilla-Colombia. De acuerdo con esto, se realizaron cuatro (4) observaciones de las variables dependientes: adherencia al tratamiento anti-retroviral y ajuste psicológico. La primera observación $\left(\mathrm{O}_{1}\right)$ se realizó dos $(2)$ meses antes de aplicar el tratamiento $(\mathrm{X})$, la segunda $\left(\mathrm{O}_{2}\right)$, tercera $\left(\mathrm{O}_{3}\right)$ y cuarta $\left(\mathrm{O}_{4}\right)$ observación se realizaron a las dos (2) semanas, tres (3) meses y seis (6) meses respectivamente luego de aplicado el tratamiento (Tabla 4).

El tratamiento $(\mathrm{X})$ consistió en la aplicación del modelo clínico "DIRÉ" de revelación del diagnóstico VIH/SIDA a niños y niñas basado en el modelo Blasini y otros ${ }^{9}$ de cinco componentes, donde se desarrollaron 16 sesiones de educación e intervención a cuidadores y niños y niñas con duración de una hora para cada familia: Las adaptaciones realizadas se explican por la necesidad de diseñar una intervención que cumpliera con las adaptaciones culturales y educativas de las familias colombianas $\mathrm{y}$, principalmente por las edades de los niños que se abordaron en este contexto, así como la necesidad de estructurar sesiones que pudieran desarrollarse en un menor tiempo debido al modelo de atención en salud vigente en Colombia. Se realizó control de variables intervinientes sobre la variable dependiente ajuste psicológico, a saber: sexo, grupo de edad, tipo de cuidador, infección por VIH/ SIDA del cuidador, escolaridad, funcionalidad familiar, auto-estima, depresión, ansiedad y funcionamiento inmunológico. Para el análisis de los datos se utilizó el paquete estadístico para las ciencias sociales SPSS versión 20. Para la variable ajuste psicológico se utilizó un análisis paramétrico mediante la prueba t-Student para muestras relacionadas y para la variable adherencia al tratamiento anti-retroviral se utilizó un análisis no paramétrico mediante la prueba de Friedman.

\section{Resultados}

No se encontraron diferencias significativas $(p<3,92)$ en la adherencia al tratamiento de niños y jóvenes, a las dos semanas, tres meses y seis meses después de la revelación con el modelo DIRÉ, cuando se comparan con los valores basales dos meses previo a la revelación y de acuerdo a la evaluación con el método indirecto de Morisky-Green-Levine. Para esta variable se concluye que la revelación del diagnóstico no tiene efectos en la disminución del cumplimiento de la terapia anti-retroviral 


\begin{tabular}{|c|c|c|c|}
\hline $\begin{array}{l}\text { Componentes del modelo } \\
\text { clínico "DIRÉ" }\end{array}$ & $\begin{array}{l}\text { n de } \\
\text { sesiones }\end{array}$ & Descripción de sesiones & Instrumentos empleados \\
\hline $\begin{array}{l}\text { 1. Seminarios de entrenamiento } \\
\text { para profesionales de la salud }\end{array}$ & Una sesión & $\begin{array}{l}\text { Capacitación a profesionales en el tema } \\
\text { de revelación del diagnóstico }\end{array}$ & Instrumento para profesionales de la salud diseñado por los autores (Trejos, 2012) \\
\hline $\begin{array}{l}\text { 2. Sesiones de intervención y } \\
\text { educación para cuidadores }\end{array}$ & $\begin{array}{l}\text { Cuatro } \\
\text { sesiones }\end{array}$ & $\begin{array}{l}\text { Explicación del protocolo y obtención del } \\
\text { consentimiento informado por parte del } \\
\text { cuidador del niño (a). Sesiones de trabajo } \\
\text { clínico para abordar temores del cuidador } \\
\text { frente a la revelación y entrenamiento } \\
\text { en cómo responder preguntas difíciles o } \\
\text { dolorosas del niño (a) }\end{array}$ & $\begin{array}{l}\text { Formulario de Consentimiento } \\
\text { Instrumento para cuidadores diseñado por los autores (Trejos, 2012) } \\
\text { Apgar familiar (Smilkstein, 1978) } \\
\text { EuroQol-EQ-5D (EuroQol Group, 1990) } \\
\text { Cuestionario MOS de apoyo social percibido (Sherbourne y Steward, 1991) } \\
\text { Cuestionario de información sobre tratamiento para VIH/SIDA de Blasini y otros } \\
\text { (2004) } \\
\text { Cuestionario de adherencia al tratamiento autoreferido de Morisky-Green-Levine } \\
\text { (Val Jiménez, Amorós, Martínez, Fernández, \& León, 1992) } \\
\text { Inventario del comportamiento de niños 6-18 años para padres (Achenbach y } \\
\text { Rescorla, 2001) }\end{array}$ \\
\hline $\begin{array}{l}\text { 3. Sesiones de intervención y } \\
\text { educación para niños y niñas }\end{array}$ & $\begin{array}{l}\text { Cinco } \\
\text { sesiones }\end{array}$ & $\begin{array}{l}\text { Obtención del perfil psicosocial del niño } \\
\text { (a). Se trabajan sesiones de dibujo de } \\
\text { agentes infecciosos antes de la revelación }\end{array}$ & $\begin{array}{l}\text { Cuestionario pre-revelación del diagnóstico de infección por VIH/SIDA diseñado } \\
\text { por los autores (Trejos, 2012) } \\
\text { Inventario de Depresión Infantil-CDI (Kovacs, 1983) } \\
\text { Inventario de Ansiedad-Estado Rasgo (STAIC) (Castrillón y Borrero, 2005) } \\
\text { Inventario de comportamiento de niños 11-18 años (Achenbach y Rescorla, 2001) } \\
\text { Material disponible para colorear de agentes infecciosos diseñado por los autores } \\
\text { (Trejos, 2012) }\end{array}$ \\
\hline 4. Sesión de revelación & Una sesión & $\begin{array}{l}\text { Construcción del modelo de virus infantil } \\
\text { desarrollado por los autores (Trejos, 2012) }\end{array}$ & Material disponible para la revelación diseñado por los autores (Trejos, 2012) \\
\hline 5. Seguimiento post-revelación & $\begin{array}{c}\text { Tres } \\
\text { sesiones o } \\
\text { más según } \\
\text { el caso }\end{array}$ & $\begin{array}{l}\text { Construcción infantil del Linfocito T CD4 } \\
\text { como soldado defensor del sistema inmu- } \\
\text { nológico diseñado por los autores (Trejos, } \\
\text { 2012). Trabajo clínico para fortalecer la } \\
\text { adherencia del niño (a) y el aprendizaje } \\
\text { de los mecanismos de transmisión de VIH }\end{array}$ & $\begin{array}{l}\text { Material disponible para el seguimiento post-revelación diseñado por los autores } \\
\text { (Trejos, 2012) } \\
\text { Material disponible para colorear de los medicamentos y mecanismos de trans- } \\
\text { misión del VIH diseñado por los autores (Trejos, 2012) } \\
\text { Administración del inventario de comportamiento de niños 11-18 años } \\
\text { (Achenbach y Rescorla, 2001) y Cuestionario de adherencia al tratamien- } \\
\text { to autoreferido de Morisky-Green-Levine (Val Jiménez, Amorós, Martínez, } \\
\text { Fernández, \& León, 1992) a las dos semanas, tres meses y seis meses post- } \\
\text { revelación }\end{array}$ \\
\hline
\end{tabular}

para el caso de los niños cumplidores. No obstante, tampoco hay evidencia estadística suficiente para afirmar que en el caso de los niños incumplidores, el conocimiento de su diagnóstico tenga como beneficio la adherencia al régimen de tratamiento establecido, aunque se observó seis meses después una reducción en $50 \%$ de los casos en la categoría de incumplidores.

Con respecto a la variable de ajuste psicológico, evaluado a través de la Lista de Síntomas del Niño CBCL/6-18, se encontraron diferencias significativas positivas en la prueba $T$ para muestras relacionadas en los seguimientos comparados con el basal: Dos semanas ( $\mathrm{p}$ : $0,001)$; tres meses (p: 0,000) y seis meses (p: 0,000) de la escala de síndrome o perfiles de síndromes empíricos (aislamiento, ansiedad/depresión, problemas psicosomáticos, problemas sociales, problemas de pensamiento, problemas de atención, conducta de romper normas y conducta de agresividad). Estas diferencias indican una disminución de los síndromes empíricos antes mencionados en los niños (as) con infección por VIH.
En relación a los efectos de normalización encontrados a las dos semanas, tres y seis meses después de la revelación del diagnóstico en la variable ajuste psicológico, entendida como una expresión clínica que contempla aspectos de irregularidad psicológica y de salud mental mediante la presencia o ausencia de problemas de tipo internalizado (ansiedad, depresión y problemas psicosomáticos) y de tipo externalizado (agresión y ruptura de normas), se puede concluir que dos meses antes de la revelación, $64,3 \%$ de los niños se encontraban en el rango normal, $14,3 \%$ en el rango borderline y $21,4 \%$ en el rango clínico. Estos porcentajes se incrementaron en las observaciones realizadas dos semanas después ubicándose $85,2 \%$ de los niños (as) con infección por VIH en el rango normal, $11,1 \%$ en el borderline y $3,7 \%$ en el rango clínico. Tres meses después de la revelación, $92,3 \%$ de los niños se ubicaron en el rango normal y 7,7\% en el rango borderline y seis meses después, $96,2 \%$ se ubicaron en el rango normal frente a $3,8 \%$ que se ubicaron en el rango borderline. 
En los resultados de la prueba $\mathrm{T}$ para muestras relacionadas de la Lista de Síntomas del Niño CBCL/6-18, controlado por sexo, no se encontraron diferencias significativas para el sexo masculino en los tres pares de observación. Para el sexo femenino se encontró una reducción de las puntuaciones a los tres y seis meses $(p<0,000$; $\mathrm{p}<0,000)$ post-revelación del diagnóstico comparados con el basal. Para el grupo de edad 6-11 años se observó una reducción de las puntuaciones a los tres y seis meses $(\mathrm{p}<0,000 ; \mathrm{p}<0,000)$ post-revelación del diagnóstico comparados con el basal. Para el grupo de edad de 12-18 años no se encontraron diferencias significativas en los tres pares de comparación post-revelación $(\mathrm{p}<0,273$; $\mathrm{p}<0,136$ y $\mathrm{p}<0,066)$.

Para los menores que se encuentran bajo el cuidado de sus madres/padres biológicos se encontró una reducción de las puntuaciones del ajuste psicológico tres y seis meses $(\mathrm{p}<0,000 ; \mathrm{p}<0,000)$ después de la revelación del diagnóstico. Del mismo modo para el grupo de niños (as) con VIH que se encuentran bajo la protección de otro cuidador diferente al biológico ( $\mathrm{p}<0,000$ para tres meses; $\mathrm{p}<0,000$ para seis meses).

En cuanto a los resultados controlados por presencia de VIH del cuidador, se observó una reducción de las puntuaciones del ajuste psicológico tres y seis meses $(\mathrm{p}<0,000$; $\mathrm{p}<0,000)$ después de la revelación del diagnóstico, tanto para el grupo de menores que se encuentran bajo el cuidado de una persona seropositiva para VIH/SIDA como para el grupo de niños con un cuidador seronegativo.

En los resultados de la prueba T para muestras relacionadas, controlado por escolaridad, se encontraron algunas diferencias significativas en los tres pares de comparación ( $\mathrm{p}<0,105 ; \mathrm{p}<0,004$ y $\mathrm{p}<0,004)$ para los niños (as) que se encuentran cursando primero y segundo de primaria. Para los niños (as) que se encuentran cursando entre tercero y quinto de primaria se encontraron diferencias significativas en los pares de comparación tres meses después $(p<0,001)$ y seis meses después $(p<0,001)$. No se encontraron diferencias significativas en los tres pares de comparación $(\mathrm{p}<0,424$; $\mathrm{p}<0,341$ y $\mathrm{p}<0,185)$ para los niños (as) que no tienen grado de escolaridad.

Respecto a los niños (as) que perciben normo-funcionalidad familiar se encontró una reducción de las puntuaciones del ajuste psicológico tres y seis meses ( $\mathrm{p}<0,000$; $\mathrm{p}<0,000)$ después de la revelación del diagnóstico. Para el caso de los niños (as) que perciben disfunción familiar moderada se encontró una reducción significativa de las puntuaciones en los tres pares de comparación ( $\mathrm{p}<0,002$; $\mathrm{p}<0,002$ y $\mathrm{p}<0,002$ ). No se encontraron diferencias en las puntuaciones en cuanto a los niveles de auto-concepto.

Para la variable sintomatología depresiva se observó una disminución en las puntuaciones del ajuste psicológico a las dos semanas, tres y seis meses $(\mathrm{p}<0,002 ; \mathrm{p}<0,000$ $\mathrm{y} p<0,000)$ después de la revelación del diagnóstico $\mathrm{y}$, finalmente también se identificó una disminución en las puntuaciones del ajuste psicológico a las dos semanas, tres y seis meses en el control realizado por ansiedad de tipo estado $(\mathrm{p}<0,001 ; \mathrm{p}<0,000$ y $\mathrm{p}<0,000)$ y por ansiedad de tipo rasgo $(p<0,001 ; p<0,000$ y $p<0,000)$.

\section{Discusión}

Teniendo en cuenta que los pacientes pediátricos ahora están viviendo más tiempo con una mejor calidad de vida, la revelación del diagnóstico de infección por VIH es un tema que debe ser considerado en los niños y adolescentes en edad escolar, en el contexto familiar e institucional. Sin embargo, la ausencia de una metodología para llevar a cabo este proceso en las instituciones prestadoras de servicios de salud en el contexto colombiano, destaca el vacío teórico frente a la descripción de los factores asociados a la reticencia de los profesionales de la salud y cuidadores a revelar el diagnóstico de infección por $\mathrm{VIH}$ a los niños en nuestro contexto ${ }^{42}$, tal como se ha venido enfatizando en varios estudios ${ }^{2,22-31,41}$ así como el desconocimiento de los beneficios ${ }^{3,9,22,23,27,28}$ que tiene el conocimiento del diagnóstico para los niños y jóvenes.

Para la variable de la adherencia al tratamiento, los resultados de esta investigación muestran que la adherencia no se ha modificado después del conocimiento del diagnóstico para los niños, lo que puede explicarse por procesos de interiorización de la información ofrecida por los profesionales de la salud y, en mayor medida, por sus cuidadores acerca del régimen de tratamiento ${ }^{32}$ (nombres, horarios y dosis) y las razones indirectas o no referidas a la enfermedad del VIH/SIDA por las que se considera importante que se tomen los medicamentos.

Los datos de este estudio sugieren que el cuidador principal tiene una responsabilidad importante en la adherencia de la terapia del menor. Estos resultados son consistentes con otros estudios ${ }^{33,34}$ que indican que la percepción de la importancia de tomar la medicación por parte del cuidador, es clave para preservar la calidad de vida de los niños y asegurar su supervivencia, debido a que lleva a implementar estrategias para motivar al niño y apoyarlo en las dificultades de la ingesta de medicamentos, principalmente relacionadas con el sabor amargo de algunos medicamentos prescritos.

En el caso de los niños y jóvenes con una buena adherencia al tratamiento, los análisis realizados para esta variable dependiente muestran que la comunicación del diagnóstico no tiene ningún efecto sobre el cumplimiento de la terapia anti-retroviral; sin embargo, no existe suficiente evidencia estadística para decir que, en el caso de los niños con dificultades presentadas en la adherencia al tratamiento, el conocimiento de su diagnóstico tenga algún beneficio para aumentar la adherencia al tratamien- 
to. No obstante, en las estadísticas descriptivas hubo una reducción de $50 \%$ de los casos de niños (as) incumplidores seis meses después de la implementación del modelo clínico "DIRÉ".

En cuanto a la variable ajuste psicológico, los resultados son consistentes con lo hallado por Blasini y otros ${ }^{9}$, sobre los efectos de la normalización en la variable de ajuste psicológico en las tres observaciones realizadas post-revelación del diagnóstico, reflejado en una disminución de las puntuaciones que indican problemas de comportamiento de tipo internalizado (ansiedad, depresión y problemas psicosomáticos) y problemas de comportamiento externalizado (agresión y ruptura de normas $)^{35-37}$

Las diferencias encontradas post-revelación del diagnóstico en el grupo de participantes del sexo femenino pueden explicarse por las diferencias en las puntuaciones del cuestionario Lista de Síntomas del Niño CBCL/6-18 para ambos sexos.

Las diferencias significativas encontradas en niños escolarizados de tercer a quinto grado en los pares de comparación tres y seis meses post-revelación, pueden indicar que el modelo clínico DIRÉ funcione mejor con niños que se encuentren recibiendo educación formal mediante la cual puedan adquirir un grado de madurez cognitiva; es bien sabido que la adquisición de conocimientos en la escuela posibilita a los niños la reorganización cognitiva, que ayuda a su progresiva comprensión y resolución de problemas a los que se enfrenten, sobre todo en el aprendizaje de la lectura y escritura que les permite a los niños ser capaces de comprender el mundo, expresar pensamientos e ideas, y comunicarse e interactuar con su mundo social ${ }^{38,39,40}$.

El modelo "DIRÉ" demostró eficacia en la normalización del ajuste psicológico y mantenimiento del cumplimiento terapéutico, favoreciendo la confianza de profesionales y cuidadores participantes en este difícil proceso. Además, otorga las herramientas a profesionales, cuidadores y niños (as) con VIH para aprender acerca del diagnóstico de una manera lúdica y adecuada al nivel de desarrollo cognitivo de cada participante, atendiendo a las directrices internacionales sobre el tema de la revelación.

Agradecimientos: Al Departamento de Investigación, Desarrollo e Innovación de la Universidad del Norte por su capacidad de respuesta y apoyo durante el desarrollo de la investigación. A la Doctora Ileana Blasini Vega, por su hospitalidad y dedicación para transmitirnos el conocimiento del proceso de la revelación del diagnóstico de VIH a los niños y jóvenes. A los equipos interdisciplinarios: Oncovihvida
IPS, IPS Medicina Integral, Santo Tomás de Villanueva Fundación Social IPS, IPS Saludcoop Costa y EPS de la confianza depositada en nuestro equipo y por ponernos en contacto con su más importante razón de ser: los pacientes y sus familias, a ellos les debemos nuestro más profundo agradecimiento. Es bien conocido el impacto del VIH/SIDA en las familias, entre las cuales es la pérdida de apoyo social y familiar, el estigma y la discriminación, y el impacto físico, psicológico y económico. A pesar de esto, las familias que participan en esta investigación enfrentaron con valor y fuerza sus temores asociados a la revelación del diagnóstico de VIH a sus niños y jóvenes.

\section{Resumen}

Antecedentes: Un estudio realizado en cinco ciudades colombianas en el 2006 ratificó lo hallado en investigaciones internacionales: los niños con infección por VIH desconocen su diagnóstico; los cuidadores son renuentes a dar esta información porque consideran que la noticia causará estrés emocional al menor, convirtiéndose en propósito principal de este estudio validar un modelo de revelación. Materiales y Métodos: Se implementó un modelo clínico, denominado: "DIRÉ", el cual, hipotéticamente tendría efectos de normalización del ajuste psicológico y adherencia al tratamiento anti-retroviral de niños con infección por VIH, mediante diseño cuasi-experimental. Se administraron pruebas (cuestionario para evaluar los patrones de revelación y no revelación del diagnóstico de VIH/SIDA a menores en profesionales de la salud y cuidadores participantes, Apgar Familiar, EuroQol EQ $-5 \mathrm{D}$, encuesta de apoyo social MOS, cuestionario de información del tratamiento para el VIH/SIDA y la Lista de Síntomas del Niño CBCL/6-18 adaptado a población latina) antes y después de la implementación del modelo a 31 niños (n: 31 ), 30 cuidadores (n: 30) y 41 profesionales de la salud (n: 41). El procesamiento de datos se realizó con el paquete estadístico para las ciencias sociales SPSS versión 21 mediante la aplicación de pruebas paramétricas (Friedman) y no paramétricas (Prueba T). Resultados: No se encontraron diferencias significativas en la adherencia al tratamiento (p: 0,392), en el ajuste psicológico se encontraron diferencias significativas positivas en los seguimientos comparados con el basal: dos semanas (p: 0,001); tres meses (p: 0,000) y seis meses (p: 0,000). Conclusiones: El modelo "DIRÉ" demostró eficacia en la normalización del ajuste psicológico y mantenimiento del cumplimiento terapéutico, favoreciendo la confianza de profesionales y cuidadores participantes en este difícil proceso. 


\section{Referencias bibliográficas}

1.- Trejos A, Tuesca R, Mosquera M. Niñez afectada con VIH/SIDA: Uso y acceso a Servicios de Salud en cinco ciudades colombianas. Revista Salud, 2011; 27 (02).

2.- Instituto Colombiano de Bienestar Familiar (ICBF), Save the Children, UNICEF y Universidad del Norte. Informe: Calidad de vida, apoyo social y utilización de servicios de salud y educación en niños, niñas y adolescentes y sus acudientes afectados con VIH/SIDA en cinco ciudades-región colombianas: Cali y Buenaventura y Barranquilla, Santa Marta y Cartagena. 2006. Universidad del Norte, Atlántico. Barranquilla: Dirección de Investigaciones y Proyectos (DIP) Universidad del Norte.

3.- Committee on Pediatric AIDS. Disclosure of illness status to children and adolescents with HIV infection. Pediatrics 1999; 103 (1): 164-6.

4.- Orcasita L, Uribe A, Valderrama L. Conocimientos y actitudes frente al VIH/SIDA en padres de familia de adolescentes colombianos. Revista colombiana de psicología 2013; 22 (01): 59-73.

5.- Almanza A, Flores F. Resistencia a la discriminación: narrativas familiares acerca de la infección por VIH. Psicología y Salud 2012; 22 (2): 173-84

6.- $\quad$ Spinetta J, Deasy-Spinetta P. Emotional aspects of life-threatening illness in children. 1980; Rockville, MD: Cystic Fibrosis Foundation. Lee y Johan.

7.- Lee C, Johann-Liang R. Disclosure of the diagnosis of HIV/AIDS to children born of HIVinfected mothers. AIDS Patient Care and STDs 1999; 13 (1): 41-5.

8.- Achucarro S. Calidad de vida de pacientes con VIH/SIDA y atención integral de enfermería. Rev Inst Med Trop 2010; 5 (1): 20-34.

9.- Blasini I, Chantry C, Cruz C, Ortiz L, Salabarría I, Scalley N, et al. Disclosure model for pediatric patients living with HIV in Puerto Rico. Develop Behavior Pediatr 2004; 25 (3): 181-9.

10.- Moriyama I. Indicators of social change. Problems in the measurents of heath status. 1968; New York: Russel Sage Foundation.

11.- Smilkstein G. The family APGAR: a proposal for a family function test and its use by physicians. J Fam Practice 1978; 1231-9.

12.- EuroQol Group. EuroQol: A new facility for the measurement of health related quality of life. Health Policy 1990; 199-208.

13.- Sherbourne C, Steward A. The MOS social support survey. Social Sci Med 1991; 32: 705-12.

14.- Achenbach T, Rescorla M. Manual for the ASEBA School-Age Forms \& Profiles. 2001; Burlington, VT: University of Vermont,
Research Center for Children, Youth and Families.

15.- Ravens-Sieberer U, Bullinger M. Questionnaire for measuring health-related quality of life in children and adolescents. Qual Life Res, 2000: 653.

16.- Piers E. Prediction on children's self-concepts. J Consult Clin Psychol 1972; 38: 428-33.

17.- Kovacs M. The Children's Depression Inventory: A self-rated depression scale for school-aged youngsters. 1983; Unpublished manuscript. University of Pittsburgh.

18.- Castrillón D, Borrero P. Validación del Cuestionario de Ansiedad Estado-Rasgo STAIC en niños escolarizados entre los 8 y 15 años de edad. Acta Colombiana de Psicología 2005: 79-90.

19.- Morisky D, Green L, Levine D. Concurrent and predictive validity of a self-reported measure of medication adherence. Med Care 1986: 67-74.

20.- Haynes R, Sackett D, Gibson E. Improvement in medication compliance in uncontrolled hypertension. 1976; Lancet.

21.- Lacalle Sisteré M. Tesis Doctoral: Escalas DSM del CBCL y YSR en niños y adolescentes que acuden a consulta en servicios de salud mental. 2009; Bellaterra: Universidad Autónoma de Barcelona.

22.- Tasker M. How can I tell you?: Secrecy and disclosure with children when a family member has AIDS. 1992; Bethesda, MD: Association for the Care of Children's Health.

23.- Lipson M. What do you say a child with AIDS. The Hastings Center Report 1993; 23: 6 .

24.- Lester P, Chesney M, Cooke M, Weiss R, Whaley P, Pérez B, et al. Factors associated with diagnostic disclosure and emotional distress in HV-infected children. J Acq Immun Def Syndr 2002; 31: 309-17.

25.- Boon-yasidhi V, Kottapat U, Durier Y, Plipat N, Phongsamart W, Chokephaibulkit K, et al. Diagnosis disclosure in HIV-Infected Thai children. J Med Assoc Thailand 2005; 88.

26.- Wiener L, Battles H, Heilman N, Sigelman C, Pizzo P. Factors associated with disclosure of diagnosis to children with HIV/AIDS. Pediatric AIDS \& HIV Infection 1996; 7: 310-24.

27.- Instone S. Perceptions of children with HIV infection when not told for so long: implications for diagnosis disclosure. J Pediatric Health Care 2000; 14: 235-43.

28.- Oberdorfer P, Puthanakit T, Louthrenoo O, Charnsil C, Sirisanthana V, Sirisanthana T. Disclosure of HIV/AIDS diagnosis to HIVinfected children in Thailand. J Paediatr Child Health 2006: 42: 283-8.

29.- Ledlie S. Diagnosis disclosure by family caregivers to children who have perinatally acquired HIV disease: when the time comes. Nurs Res 1999; 48: 141-9.

30.- Waugh S. Parental views on disclosure of diagnosis to their HIV-positive children. AIDS Care 2003; 15 (2): 169-76.

31.- Kouyoumdjian F, Meyers T, Mtshizana S. Barriers to disclosure to children with HIV. J Trop Pediatr 2005: 51-5.

32.- Trejos A, Mosquera M, Tuesca R. Niñez afectada con VIH/SIDA: Calidad de vida, funcionalidad familiar y apoyo social en cinco ciudades colombianas. Rev Salud Uninorte 2009; 25 (01): 17-32.

33.- Marthefka S, Tepper V, Brown J, Farley J. Caregiver psychosocial characteristics and children's adherence to antiretroviral therapy. AIDS Patient Care STDs 2006; 20 (6): 429-37.

34.- Hammami N, Nöstlinger C, Hoerée C, Lefévre P, Jonckheer T, Kolsteren P. Integrating adherence to highly active antiretroviral therapy into children's daily lives: A qualitative study. Pediatrics 2004; 114: 591-7.

35.- Achenbach System of Empirically Based Assessment. Manual for the child behavior cheeklist 4-18 and 1991 profile. 1991; Burlingtong, VT: University of Vermont: Department of Psychiatry.

36.- Achenbach T, Rescorla M. Manual for the ASEBA School-Age Forms \& Profiles. 2001; Burlington, VT: University of Vermont, Research Center for Children, Youth and Families.

37.- Piaget J. Seis Estudios de Psicología. 1985; Barcelona: Planeta-gostini.

38.- Bibace R, Walsh M. Development of children's concept of illness. Pediatrics 1980; 66: 912-7.

39.- Martín L, Grau J, Espinosa A. Conceptual framework for evaluating and improving adherence to medical treatment in chronic diseases. Rev Cubana Salud Pública 2014 Jun [citado 2015 mayo 27]; 40 (2): 222-35. Disponible en: http://scielo.sld. cu/scielo.php?script $=$ sci_arttext\&pid $=$ S086434662014000200007\&lng=es.

40.- Varela M T, Gómez O L, Mueses H F, Galindo J, Tello I. Factores relacionados con la adherencia al tratamiento farmacológico para el VIH/SIDA. Salud Uninorte. Barranquilla (Col.) 2013; 29 (1): 83-95.

41.- Trejos A M, Reyes L, Alarcón Y, Bahamon M. Patterns of HIV status disclosure and nondisclosure to children under 17 years in health professionals and caregivers participants in "DIRÉ" clinical model in BarranquillaColombia. Pensee J 2014; 76: 148-60.

42.- Trejos A M, Reyes L, Alarcón Y, Bahamon M. (2015) ¿Tienen el derecho los niños (as) seropositivos para VIH/SIDA a conocer su diagnóstico?: La Revelación a través del Modelo Clínico "DIRÉ”. En Y. Alarcón, F. Vásquez, W. Pineda e Y. Martínez (Eds). Estudios actuales en Psicología (pp: 223-241). Barranquilla: Ediciones Universidad Simón Bolívar. 